\section{CYCLONES ON THE SUN.}

Important discoveries have been made during the past few months at the Carnegie Solar Observatory in California, where Prof. George E. Hale, its director, has gained valuable knowledge about the sun in showing the process whereby fuel is fed into the solar furnace. By improved methods of research, by careful diligence in closely observing the sun, aided by a little stroke of luck, photographs have been taken which show a mass of cool hydrogen gas being sucked into the vortex of a sun spot, the result of a terrific solar cyclone. These critical photographs, which are of excellent quality, speak volumes for the observers on Mount Wilson in regard to their ability in perfecting new lines of research, their hard-working methods in observing and their genius in properly interpreting the results of these fine pictures. When related, these discoveries appear extremely sim. ple and matter-of-fact, but the work that has led up to them required a series of brilliant discoveries which are probably equaled nowhere in any other science.

Mount Wilson near Pasadena in California was chosen for the observatory of the Carnegie Institution because its position of fered the possibility of continuously observing the heavens for weeks at a time unhampered by clouds. The talented director of the Yerkes Observatory of the University of Chicago, chosen as its head, decided to limit observational work to the sun, for the reason that the sun is a typical star, and in closely studying it we are not only gaining information about the body which is of most importance to us on earth, but we are also shedding considerable light on the great and im portant problem of astronomy, the study of stellar evolution. While still a young man in 1893 , Prof. Hale invented the spectroheliograph, and since that time much has been expected from him in astronomic research. His present discoveries are a fitting cri$\max$ to a long series of brilliant discoveries rendered possible by great genius and remarkable enthusiasm.

If astronomers of the present day had only the photographic camera to assist with their eye observations at the end of a telescope, very little could be kne photographic plate has been of enormous value in giving a permanent record of fleeting phenomena on the sun's surface, and without it the great advances of to-day would have been impossible. Since 1868 the spectroscope has given its aid to solar investigations. In that year Janssen of France and Lockyer of England almost simultaneously pointed out that the red flames or prominences, which formerly could be seen only during the few minutes of a total solar eclipse, were now an everyday phenomenon. By a peculiar use of the spectroscope it is now possible to see these red flames in broad daylight when they are on the sun's edge, in spite of the fact that the light from the sun is a thousandfold more powerful than the light from the prominences. Another from the prominences. Another capable hands of the late Prof. Young of Princeton, a long list of discoveries were added to the scienc of astrophysics. Flames could be seen shooting to enormous distances from the sun's edge at a speed greater than one hundred miles per second! The end of all research seemed nearly reached when it became possible to photograph and obtain a permanent record of these gigantic eruptions, as was possible by Prof. Hale's invention of the spectroheliograph. A the name signifles, the sun is photographed by mean of its spectrum. A powerful grating or prism train at the eye end of the telescope spreads the sun's light out into its spectrum. By allowing the light from one line of the spectrum, as $H$ or $K$, to pass through a secondary slit, a photograph of the sun and its surroundings can be taken in $\boldsymbol{E}$ or $\boldsymbol{K}$ light alone. Over- coming the mechanical dificulties involved in this, gave Prof. Hale the spectroheliograph. At flrst on his 12-inch - telescope, later on the 40-inch of the Yerke Observatory, were photographed wonderful pictures of the prominences at the sun's limb, the sun on the original plates with the 40-inch being seven inches in diameter. With the new spectroheliograph it became possible to photograph not only the prominences but also the face of the sun, and if the secondary slit were set at the center of the $K$ line at the violet end of the spectrum, a plate of the sun was obtained in the light of glowing calcium vapor. This photograph wa decidedly different in appearance from that of a straight picture taken in the ordinary way.

In experimenting with the spectroheliograph, it wa found that the solar image looked different according as the second slit was set directly on the center of the $K$ line or a little to the red or tho violet side. tage of a location in California was recognized by the Carnegie Institution. Work there progressed along the same lines which had brought so much success at the Yerkes Observatory; and Prof. Hale planned to carry out researches on a grander scale than was possible even with the 40-inch telescope.

In carrying out the work on the fiocculi, the greatest success had been obtained in the use of the calcium $H$ and $K$ lines of the solar spectrum. The hydrogen lines $H \beta, H \gamma$, and $H \delta$, were also used to photograph these interesting phenomena, and it was found that there were great differences in the appearance of the sun as the calcium lines were employed or as the hydrogen lines were made use of. In fact, there were marked variations as the sun was photographed in the light of each of these three hydrogen lines. Consequently, Prof. Hale desired greatly to learn what would be the results when the $\mathrm{Ha}$ line of hydrogen was employed. The $H a$ or the $C$ line of the solar specor the $C$ line of the one exclusively used in visual observations of prominences. But unfortunately this $C$ line is far in the red, and the ordinary photographic plate is not sensitive to red light a fact of which we are all aware, since photographs are ordinarily developed in red light. If a plate sensitive to red light could be obtained which would not require too long an exposure, it might then be possible to photograph the sun in the light of glowing hydrogen gas the $C$ line. Experiments to make red-sensitive plates rapid enough for use- had not met with much success until within the last year. Mr. Robert James Wallace of the Yerkes Observatory showed a method of doing this by bathing ordinary plate ordinary plates in a certain solution. With this new weapon of research photographic work on the sun was undertaken at Mount Wilson with renewed energy. Seed's "Gilt Edge" plates bathed according to Wallace's directions proved to be splendidly adapted to the purpose. The first experiments with the new plates wer tried about the middle of March 1908 , though the flrst satisfactory photograph was that of April 30 . The new plates showed a great improvement in detail over the old. Not only were prominences seen better, but the spots on the sun's surface took on an added interest, for it was at once seen that they are centers of attrac tion which draw toward them the hydrogen of the solar atmosphere. It became necessary now more closely than ever to observe sun spots, for discoveries would follow each other in rapid order.

On May 26, 1908, a spot reached the east limb of the sun at $8: 16$ A. M. and the looked-for opportunity was at hand. On May 25 , before the spot turned the edge of the sun, evidences of activity could be seen in the shape of prominences which were undoubtedly connected with the spot group. On May 28 at 6:58 A. M. with the spot very close to the eastern limb, traces of a cyclone could be seen near the spot, matter there being in rapid whirling motion, and likewise was seen what proved later to be especially interesting, a flocculus of dark cool hydrogen. (The spot re-
(The sun until June 8.) The

CYCLONES ON THE SUN.

This had led to the discovery of flocculi by Prof. Hale, masses of luminous gas floating at different elevations above the sun's surface. Still later investigations led Prof. Hale to the conclusion that it was now possible to take photographs representing the condition of the sun's atmosphere at different elevations. When we think that remarkably little is known of our own atmosphere at a height of flve miles above the earth's surface, we get a slight idea of the power of the astronomer in photographing the sun ninety-three millions of miles away, and gaining a knowledge of the condition of the sun's atmosphere at different levels! This work was of far-reaching importance and it became more and more necessary to photograph the sun daily. But at Yerkes and at all observatories in the eastern part of our country, daily work on the sun is often interrupted by clouds, and the adran. mained on the face of the sun until June 8.) The
splendid series of photographs taken show the cyclones splendid series of photographs taken show the cyclones
continuing on a gigantic scale around the spot. The continuing on a gigantic scale around the spot. Th
dark mass of hydrogen-the flocculi-showed change here and there, giving evidence of great agitation on the sun. Suddenly on June 3 a catastrophe happened; the cool hydrogen gas, which had been continuously in the same location since the spot came around the edge of the sun on May 26, was quickly set whirling and was rapidly sucked into the great maelstrom on the sun. Prof. Hale was lucky enough to have this great solar cyclone-the flrst of its kind ever seenrecorded on a series of nine photographs all taken within ten minutes. We congratulate Prof. Hale on his energy and his great fortune. The speed at which this cool hydrogen rushed into the center of the spot was about sirty miles per second-rather faster than 
any motions we know of on the earth-a velocity comparable with that at which prominences are thrown off from the sun.

The photographs showing the solar cyclone were taken about 5 P.M. June 3 . At 6 the next morning quite a change was evident around the spots. Bright masses of heated hydrogen gas began to appear, and this heated gas kept on increasing in amount for the next couple of days. Thus we become aware of the manner in which relatively cool matter is subjected to the heat of the solar furnace, tracing a mass of hydrogen gas before and after being treated by the solar fire. These are the beginnings of a new set of solar fire. These are the beginnings of a new set of
discoveries which will give us much knowledge regarding our great sun. Spots are thus the centers of disturbances on the sun. But how much disturbance is centered there? And what connection have sun spots with other phenomena? The scientific world has Innown for more than fifty years that sun spots closely affect the amount of terrestrial magnetism; spots on the sun are connected with "electric storms," and with displays of northern lights, but how: related? The sun is evidently the seat of a great electro-magnetic field, whose lines of force stretch outward even as far as the earth. In fact, the appearance of the hydrogen flocculi on the new photographs of Prof. Hale recall to the mind the appearance of iron filings in a magnetic field; and one wishes to know what kind of an electro-magnet the sun is, how it displays its force, and whether sun spots are the centers of lines' of force running out. We shall expect Prof. Hale to tell us whether the lines of the spectrum of a sun spot show the Zeeman effect, and how it is that the lines of force from the spot alter the appearance of the spectrum lines.

These discoveries emphasize strongly the great im portance of a close and careful study of sun spots, for they are somehow connected with a variety of phenomena such as electric storms, aurora borealis, prominences, flocculi, faculæ, corona, and possibly with conditions of temperature or weather here on with conc.

\section{More Curions Facts About Numbers,
BY J. F. springer.}

Those who read attentively the writer's article on numbers in the Scientific American for March 28, 1908, may have wondered how such groups of numbers as $(3,4,5),(5,12,13),(8,15,17)$, and $(7,24,25)$ may be obtained. These groups have the property that if their members be squared the sum of the first two squares is equal to the third. Thus $3^{2}+4^{2}=5^{2}$. These few cases were, perhaps without exception, found by observation. But the writer has devised a method whereby an unlimited number of such groups may be obtained with quickness and certainty. Consider the equation

[1] $\left(x^{2}+y^{2}\right)^{2}=(2 x y)^{2}+\left(x^{2}-y^{2}\right)^{2}$.

Now this equation is true irrespective of the particular values that $x$ and $y$ may have. This may be seen by actually carryinz out the operations indicated. Thus, squaring as directed, we have,

$$
x^{4}+2 x^{2} y^{2}+y^{4}=4 x^{2} y^{2}+x^{4}-2 x^{2} y^{2}+y^{4} \text {. }
$$

Returning now to equation [1], and remembering that it is true whatever the values of $x$ and $y$, we may obtain groups of numbers having the desired property by simply selecting any values we choose for $x$ and $y$. Thus, by making $x=2$ and $y=1$, we obtain ' (using dot for multiplication)

$$
\left(2^{2}+1^{2}\right)^{2}=(2.2 .1)^{2}+\left(2^{2}-1^{2}\right)^{2} \text {. }
$$

That is, $5^{2}=4^{2}+3^{2}$. This $\mathrm{i}$ i one of our old groups. Making $x=5$ and $y=2$, we obtain

yielding

$$
\left(5^{2}+2^{2}\right)^{2}=(2.5 .2)^{2}+\left(5^{2}-2^{2}\right)^{2}
$$

$29^{2}=20^{2}+21^{2}$

Or; if $x=7$ and $y=4$

$$
\begin{aligned}
& 73^{2}=48^{2}+55^{2} \text {. } \\
& \text {. }
\end{aligned}
$$

Finally, if $x=13$ and $=56^{2}+33^{2}$$$
\text { and } y=8 \text {, } 233^{2}=208^{2}+105^{2}
$$

By performing the operations of squaring, one may convince himself that these results are correct. Thus, in the last case $233^{2}=54,289$. The squares of 208 and 105 are 43,264 and 11,025, which upon being added yield 54,289. The reader should use formula [1] and devise groups for himself. By carrying out the squaring operations he may satisfy himself of the correctness of his work.

An interesting property of numbers is the fact that if we add the squares of two consecutive numbers together, we shall obtain a new number such that if we multiply it by 2 and subtract 1 , we shall obtai a square number. Thus, suppose we add the squares of the consecutive numbers 2 and 3 . Multiplying th new number (13) by 2 and subtracting 1 , we get $25-$ which is a square number. Again, adding the square of the consecutive numbers 12 and 13 , we get 313 . Multiplying this by 2 and subtracting 1 , we obtain 625-a square number. Indeed, the square numbe obtained finally is the square of the sum of the tw consecutive numbers. Thus, in the first case, 25 is the square of 5 , which is the sum of 2 and 3 . So also, 625 is the square of 25 , which is, in turn, the sum
of 12 and 13 . If we desire to see the reason for this, of 12 and 13 . If we desire to see the reason for this, $x$ and $x+1$. Squaring and adding, we obtain $x^{2}+(x+1)^{2}$, which equals $2 x^{2}+2 x+1$. Multiplying this by 2 and deducting 1 , we get $4 x^{2}+4 x+1$

But this is a square number, being in fact,

This may be written $(2 x+1)^{2}$

$$
[x+(x+1)]^{2}
$$

from which it may be seen that it is the sum of $x$ and $x+1$ which is squared. That is to say, by adding the square of the two consecutive numbers, multiplying the result by 2 and subtracting 1, we get the square of the sum of the consecutive numbers.

In the preceding article, a method was given whereby any cube could be expressed as the difference between two squares. That method utilized the formula

$$
\text { [2] } \quad x^{3}=\left(\frac{x^{3}+2}{2}\right)^{2}-\left(\frac{x^{4}-x}{2}\right)^{*}
$$

Anothēr method will now be shown, by which we may differently express a cube as the difference between two squares:

Assuming

$$
x^{3}=y^{2}-z^{2},
$$

we have to determine $y$ and $z$. Instead of factoring $x^{5}$ into $x$ and $x^{2}$ (as in the former article), we factor Into 1 and $x^{3}$. Putting these equal to the factors of $\boldsymbol{y}^{2}-\boldsymbol{z}^{2}$, we have,

$$
\begin{aligned}
& y-z=1 \\
& \text { and } y+z=x^{3}
\end{aligned}
$$

and we obtain for $y$ and $z: y=\frac{x^{3}+1}{2}, z=\frac{x^{3}-1}{8}$.

We may now write,

$$
\text { [8] } \quad x^{3}=\left(\frac{x^{3}+1}{2}\right)^{\prime}-\left(\frac{x^{3}-1}{2}\right)^{\prime} \text {. }
$$

This equation is true, whatever the value of $x$, as may be readily shown by performing the operations indicated. As formula [3] stands, it is applicable only to odd values of $x$. For, if $x$ is even, then $x^{3}$ is also even. This would make $x^{3}+1$ and $x^{3}-1$ odd numbers. Consequently $\frac{x^{3}+1}{2}$ and $\frac{x^{3}}{2}-1$ would remain fractions. If $x$ be taken equal to 13 , we shall have

$13^{3}=\left(\begin{array}{c}15^{3}+1 \\ 2^{-}\end{array}\right)^{2}-\left(\frac{13^{3}-1}{2}\right)^{\prime}$
That is $13^{3}=1,099^{2}-1,098^{2}$. Again,

That is $\begin{aligned} & 13^{3}= 1,099^{2}-1,098^{2} \text {. Again, } \\ & 7^{3}=\left(\frac{\gamma^{3}+1}{2}\right)^{2}-\left(\frac{7^{3}-1}{2}\right)^{2}\end{aligned}$

$$
\text { or, } \quad 7^{3}=172^{2}-171^{2}
$$

As formula [2] is good for all numbers, whether even or odd, it will be interesting to express by its means the cubes last used $\left(13^{3}\right.$ and $\left.7^{3}\right)$ as the difference between two squares.

$$
\begin{aligned}
\text { Thus, } & 13^{3}=\left(\frac{13^{2}+13}{2}\right)^{2}-\left(\frac{13^{3}-13}{2}\right)^{2} \\
\text { And } & 7^{3}=\left(\frac{7^{2}+7}{2}\right)^{2}-\left(\frac{7^{2}-7}{2}\right)^{2}
\end{aligned}
$$

These give, $13^{3}=91^{2}-78^{2}$

$7^{3}=28^{2}-21^{2}$

An interesting property of the natural numbers is that if we add any number of these together beginning with unity, double the sum and subtract the last of the numbers, we shall have the square of this final number. Thus, suppose we consider the numbers from 1 to 9 . Adding, $1+2+3+4+5+6+7+8+9$, we get 45. Doubling this and subtracting the final number (9), we get 81 , which is the square of the final number. To understand the reason for this is not difficult. Thus, we write

$$
s=1+2+3+4+5+6+7+8+9
$$

And, $s=9+8+7+6+5+4+3+2+1$

Adding we get $2 S=9(9+1)$

Expressed more generally, $n$ being both number of terms and last term, we have

We have therefore

$$
2 S=n(n+1)=n^{2}+n .
$$

$$
2 s-n=n^{2}+n-n=n^{2} \text {. }
$$

We will now pass to some theorems which, so far as the writer is aware, appear now for the first time. The first theorem is to the effect that if we have the fifth power of any number (not itself a multiple of 11), we may, by the addition or subtraction of unity, make this number divisible by 11 . For instance, the fifth power of 921 is $662,671,283,348,601$. By adding 1 , it is divisible by 11 . Search as you may, you will find no number (unless it itself is a multiple of 11) which upon being raised to the fifth power will not become divisible by 11 upon the addition or subtraction of 1 .

In order to establish this result generally, let us represent any number (not containing 11 as a factor) by $x$. Now this number $x$ upon being divided by 11 , will yield a remainder less than 11 and more than 0 (as it is supposedly not divisible by 11). We may write, therefore,

$x=11 y+z$,

where $z$ may have any integral value from 1 to 10 (inclusive). We now write,

$$
x^{5}=(11 y+z)^{5}
$$

$x^{5}=11^{5} y^{5}+5.11^{4} y^{4} z+10.11^{3} y^{3} z^{2}+10.11^{2} y^{2} z^{3}$ $+5.11 y z^{4}+z^{5}$

If we attentively observe the right-hand side of this equation, we shall see that 11 occurs in all the terms except the last. We may write, then,

[4] $\quad x^{5}=11 . R+z^{6}$

Now, as before observed, $z$ is limited in its range of vet us examine these

$$
\text { For } \begin{array}{rll}
z=1, & z^{5}=1 & =11 \times 0+1 \\
z=2, & z^{5}=32 & =11 \times 3-1 \\
z=3, & z^{6}=243 & =11 \times 22+1 \\
z=4, & z^{5}=1,024 & =11 \times 93+1 \\
z=5, & z^{5}=3,125 & =11 \times 284+1 \\
z=6, & z^{5}=7,776 & =11 \times 707-1 \\
z=7, & z^{5}=16,807 & =11 \times 1,528-1 \\
z=8, & z^{6}=32,768=11 \times 2,979-1 \\
z=9, & z^{5}=59,049=11 \times 5,368+1
\end{array}
$$

It will be seen, from an examination of the last column, that $z^{5}$ may always be expressed as a multiple of 11 plus or minus 1 . That is, we may write $z^{5}=11 . M \pm 1$.

Consequently, equation [4] may now be written $x^{5}=11 . R+11 . M \pm 1$

From which is easily obtained,

$$
x^{5} \mp 1=11 .(R+M) \text {. }
$$

That is to say, either the addition or subtraction of unity will cause any fifth power to become divisible by 11, unless the number raised to the fifth power is itself a multiple of 11 , when the addition or subtraction of no number is needed to make it divisible by 11 .

This seems to be a very curious result. Another apparently new proposition affirms that if we subtract a number from its seventh power the result is divisible by seven. Thus 128 is the seventh power of 2 . Subtracting 2 , we get 126 , which is divisible by 7 . Again 279,936 is the seventh power of 6 . Subtracting 6 , we obtain 279,930, which upon trial we find to be divisible by 7. As a final illustration, consider the number $62,748,517$. This is the seventh power of 13 . Subtracting 13 from it, we get $62,748,504$, which is divisible by 7 .

In order to establish this proposition generally, let $x$ represent any number not divisible by 7 . It is superfluous to consiner multiples of 7 , as the proposition is obviously true in their case. So then, we have to prove that

$$
x^{7}-x
$$

is divisible by 7 , when $x$ is a non-multiple of 7. As $\pi$ is not divisible by 7 , we may write

$x=7 y+z$, when $z$ varies from 1 to 6 (inclusive). We have, then,

$$
x^{7}-x=(7 y+z)^{7}-(7 y+z) .
$$

Now, upon expanding the right-hand side of this equation the number 7 appears in every term except $z^{7}$ and $-z$. So that we may write

$$
\text { [5] } \quad x^{7}-x=7 . Q+z^{7}-z \text {. }
$$

Let us consider the possibilities of $z^{\tau}-z$ for the six possible values of $z$ ( 1 to 6 ).

$$
\text { For } \begin{array}{rrr}
z=1, & z^{7}-z= & 0 \\
z=2, & z^{7}-z= & 126 \\
z=3, & z^{7}-z= & 2,184 \\
z=4, & z^{7}-z=16,380 \\
z=5, & z^{7}-z=78,120 \\
z=6, & z^{7}-z=279,930
\end{array}
$$

Examining the column on the right hand, we find every number to be divisible by 7 . Consequently, whatever value it is possible for $z^{7}-z$ to possess, each and every one is divisible by 7 . So that the right-hand side of equation [5] is divisible by 7, throughout-which establishes the proposition.

A similar proposition, and one apparently new, is to the effect that if we subtract a number from its thirteenth power the resulting number is divisible both by 7 and 13, and consequently by 91 (since $7 \times 13=91$ ). As an example, consider the thirteenth power of 2. This is 8,192. Subtracting 2, we obtain 8,190 , a number divisible both by 7 and 13 . Again, the number $2,541,865,828,329$ is the thirteenth power of 9 Subtracting 9, we have, 2,541,865,828,320. This number, upon trial, we find to be divisible both by 7 and 
The entire right-hand side of this equation contains 13 as a factor except

$z^{13}-z$.
Let us consider the possible values of this expression.

$$
\begin{array}{rlr}
\text { For } z=1, & z^{13}-z= & 0 \\
z=2, & z^{13}-z= & 8,190 \\
z=3, & z^{13}-z= & 1,594,320 \\
z=4, & z^{13}-z= & 67,108,860 \\
z=5, & z^{13}-z= & 1,220,703,120 \\
z=6, & z^{13}-z= & 13,060,694,010 \\
z=7, & z^{13}-z= & 96,889,010,400 \\
z=8, & z^{13}-z= & 549,755,813,880 \\
z=9, & z^{13}-z= & 2,541,865,828,320 \\
z=10, & z^{13}-z= & 9,999,999,999,990 \\
z=11, & z^{13}-z= & 34,522,712,143,920 \\
z=12, & z^{13}-z=106,993,205,379,060
\end{array}
$$

xamining the column of figures at the right hand, it will be seen that each and every number is divis ible by 13 . Consequently, the expression $x^{13}-x$ is divisible by 13 . We have yet to show that it is also divisible by 7 . We represent $x$ now by $7 m+n$, where may be any number from 1 to 6 . The expression $x^{13}-x$ now becomes

$$
x^{13}-x=(7 m+n)^{13}-(7 m+n)
$$

Just as before, every term on the right-hand side when expanded, contains the factor 7 except the expression

\begin{tabular}{|c|c|c|c|c|c|c|}
\hline \multirow{2}{*}{ 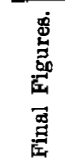 } & \multicolumn{6}{|c|}{ Powers. } \\
\hline & 1 & 2 & 3 & 4 & 5 & 6 \\
\hline $\begin{array}{l}1 \\
2 \\
3 \\
4 \\
5 \\
6 \\
7 \\
8 \\
9 \\
0\end{array}$ & $\begin{array}{l}1 \\
2 \\
3 \\
4 \\
5 \\
6 \\
7 \\
8 \\
9 \\
0\end{array}$ & $\begin{array}{l}1 \\
4 \\
9 \\
6 \\
5 \\
6 \\
\mathbf{6} \\
\mathbf{9} \\
\mathbf{4} \\
1 \\
0\end{array}$ & $\begin{array}{l}1 \\
8 \\
7 \\
4 \\
5 \\
6 \\
3 \\
2 \\
9 \\
0\end{array}$ & $\begin{array}{l}1 \\
\mathbf{6} \\
1 \\
\mathbf{6} \\
\mathbf{5} \\
\mathbf{6} \\
1 \\
\mathbf{6} \\
1 \\
0\end{array}$ & $\begin{array}{l}1 \\
2 \\
3 \\
4 \\
5 \\
6 \\
7 \\
8 \\
9 \\
0\end{array}$ & $\begin{array}{l}1 \\
4 \\
9 \\
6 \\
5 \\
6 \\
9 \\
4 \\
1 \\
0\end{array}$ \\
\hline
\end{tabular}

$$
n^{13}-n
$$

To examine this for values from 1 to 6 , is simply to look over the column of figures last obtained beginning with the first and extending through the sixth, to see whether 7 is a factor. Upon examination, we find that it is. So that as a result of our investigation we find that all numbers of the form $x^{13}-x$ ar divisible both by 7 and by 13 -consequently by $7 \times 13$ or 91.

But we have not reached the end as yet. In rais ing numbers to the several powers, we find that powers of numbers ending in 1 also end in 1 . Thus, any power of 31 will terminate in the figure 1 . If a num ber ends in 2, the final figure of its various power runs through the cycle $2,4, \overrightarrow{8}, 6$. Constructing a table, we find the following results:

Thus for numbers ending in 7, the final figures of the powers run through the cycle 7, 9, 3,1. Upon examining the table closely, it will be seen that these cycles consist of one, two, or four numbers. In consequence of this periodicity, the thirteenth power of any number will terminate in the same figure as the first power, all the cycles starting afresh at this powe (as also at the fifth and ninth). Consequently, if we subtract the first power from the thirteenth we shall obtain a number whose final figure is 0 - the final figures of minuend and subtrahend being the same. Consequently, all numbers of the form $x^{13}-x$ terminate in 0 , and are for this reason divisible by both 2 and 5 . We learn, then, that all numbers of the form $x^{13}-x$ are divisible at once by $2,5,7$, and 13 -that is, by 910.

\section{The Current Supplement.}

The opening article of the current Supplement, No 17.16, deals with the subject of the short-distance transportation of the products of the mine, field, and forest from the outlying districts to the railroad. The use of dry air in the blast furnace as patented by Gayley is described fully. Various conjectures hav been made as to the cause of the destruction of Count Zeppelin's airship. One very plausible explanation ascribes the catastrophe to the effect of atmospheric electricity. The electrical principles involved are described in the Supplement. Whether as a toy, as a scientific instrument, or as an object of practical utility, the gyroscope is of great interest. As an illus tration of its possible development, a gyroscope in vented by Prof. Ach of Berlin is described, which has for its object a means of securing the invariability of the axis of rotation of the gyroscope, no matte what arbitrary movements may take place in the carrier of the gyroscope. The invention comprises means to do away with the influence of gravity on the spinning body. Sir William Ramsay discusses the subject, "Do the Radioactive Gases Belong to the Argon Series?" The making of artificial diamonds is described. Mr. Alexander A. Chenoweth writes on reinforced concrete as a substitute for wooden piles, cross- ties, or sleepers. Photographic bird's eye views havo Dr. Niscovered that they may also be apparatus is described and illustrated.

\section{A $\$ 500$ Prize for a Simple Explanation of the Fourth}

\section{Dimension.}

A friend of the ScIFNTIFIC AMERICAN, who desires to remain unknown, has paid into the hands of the publishers the sum of $\$ 500$, which is to be awarded as a prize for the best non-mathematical explanation of the Fourth Dimension, the object being to set forth in an essay the meaning of the term so that the ordiary lay reader can understand it.

Competitors for the prize must comply with the following conditions:

1. No essay must be longer than 2,500 words

2. The essays must be written as simply, lucidly, nd non-technically as possible.

3. Each essay must be typewritten and identified with a pseudonym. The essay must be inclosed in a plain sealed envelope, bearing only the pseudonym. With the essay should be sent a second plain sealed envelope, also labeled with the pseudonym, and containing the name and address of the competitor. Both these envelopes should be sent to "Fourth Dimension Editor, Scientific American, 361 Broadway, New York, N. Y."

4. All essays must be in the office of the Scientific AMERICAN by April 1, 1909 .

5. The Editor of the Scientific American will retain the small sealed envelope containing the address of the competitor and forward the essays to a Board of Judges, who will select the prize-winning essay.

6. As soon as the Board of Judges have agreed upon the winning essay, they will notify the Editor, who will open the envelope bearing the proper pseudonym and containing the competitor's true name. The competitor will be notified by the Editor that he has won he prize, and his essay will be published in the ScIENTific American.

7. The Editor reserves the right to publish in the columns of the Scientufic American or the Scientific American Supplement three or four of the more meritorious essays, which in the opinion of the judges are worthy of honorable mention.

The Editor of the Scinvirio Amenionr is now engaged in selecting the judges who will award the prize. They will be three in number, and all will be eminent American mathematicians. The names of the judges will be announced in a later issue of this journal.

Substitute tor Chromate of silver.

Running short of Lord Kelvin's glass tubes for deepsea soundings, and finding the prices charged in America-40 cents each-rather high, Mr. John Martin of the S. S. "Akershus" informs us that he tried to find some substitute for chromate of silver, and succeeded. He bought a $1 / 4$-inch (inside diameter) watergage glass of the same length as Lord Kelvin's tubes, closed one end with a flat piece of cork, a small rag over it and some sealing wax. Next he cut a sheet of drawing paper into narrow strips at a bookbinder's. Along the middle of the paper strip he drew a line with a copying pencil. The paper slip was then shoved up the glass tube, forming a blank scale. The lower end of the strip was turned around the edge of the tube and tied with a. piece of cotton thread to prevent the paper from being displaced by the water as it rose in the tube. The tube was used in the same way as Lord Kelvin's. The depth was read off from the boxwood scale. The ink pencil line distinctly indicated how far the water reached. The guard tube containing. the gage glass was made from an old condenser tube.

\section{The Death of Wullam Edward Ayrton.}

William Edward Ayrton, the distinguished English. Wectrical engineer and inventor, died in London on November 8 in his sixty-first year. He was educated at. University College, London, and after graduation entered the Indian Government Telegraph Service. In 1873 he was appointed to the chair of natural philosophy and telegraphy at the Imperial College of Engineering, Japan. Here he remained for five years. In 1884 he was made professor of electrical engineering at the Central Technical College, South Kensington. He was president of the Physical Society, 1891-2, and president of the Institution of Electrical Engineers in 1892 .

\section{The Death of John Henry Mills.}

John Henry Mills, a pioneer heating engineer and nventor of heating boilers, of Boston, Mass., died at his home in Faneuil, a suburb of that city, on Tuesday, October 6, in his seventy-fifth year. He was n authority on heating, and his work, "Heat-Its Application to the Warming and Ventilation of Buildings," is one of the standard books on the subject.

Coxtespondente.

USEFUL ARTIFICIAL WATERWAYS.

To the Editor of the Scientific American: The proposition to retain water at the heads of large ful consideratir tributaries is surely well worth carenot only on the surface, but to great depths, replenish ing old-time underground rivers and furnishing wate for wells in the surrounding country. Such reservoir can be used to keep machinery running during the dry to power plants, by steadying business, in regulation to power plants, by steadying business, in regulating whole year.

Yet still all the water that can be -retained will prove fow of the lower streams of an checking the overers, such as the Mississippi and the Amazon.

The best possible command of the Missis can be secured by dredging a deep and broad channel for several hundred miles inland at a proper distance from the river channel on both sides of the river.
The channel on the west side should extend from
Atchafalaya Bay, keeping west of the extremely trouAtchafalaya Bay, keeping west of the extremely troublesome Bayou Lafourche and passing east of Opelouto the head of White River, across the line of Mis sippi River, and Van Buren on the Iron Mountain Railway. This will drain large tracts of wet, unproductive
flat lands, which will be of excellent quaıty and most
of them fertile and productive when properly drained of them fertile and productive when properly drained. There are hundreds of thousands of acres of flat, wet land along the proposed course of this west side canal.
The east side channel should begin at Bay St. Louis The east side channel should begin at Bay St. Louis,
extending north, crossing the Pearl River, to a poin extending north, crossing the Pearl River, to a poin cross the Illinois Central Railway, near the town of below. Natchez, on into the Yazoo Valley then on up that valley, passing near and east of Memphis and on to the Ohio River Valley. There bending east follow the course of that river as far as the.land formation makes it desirable. At certain po: its, it may 'be, neces sary to leave off one side of a river and take up con-
struction on the other side. This being a question of struction on the other side. This being a
easy decision for any competent engineer.

These channels will have a threefold purpose of use fulness: First, as channels of drainage, they will add country traversed, by providing several outlets for drainage of swamps and farm land in general. For such an outlet there is a burning necessity which should have been provided for long years ago. Second, for relieving the strain upon the levees of the Mississippi and the other rivers and bayous in cluded in the districts traversed by the canal's, also to
prevent overflow by letting surplus water into these prevent overflow by letting surplus water into these
channels, through intersecting canals a any point of greatest strain. This can be done at any point Third, if set free to the traffic of all who own suit able crafts, the value for purposes of navigation suitwill eventually. pay the cost of construction and fat dividends besides

These main channels can be: constructed a libera width in the beginning and shallow, to be deepened by dredging until, if found desirable, seagoing vessels of the highest tonnage can pass each other with ease, On the east side of the Mississippi River, beginning about 30 miles above Cairo and extending up to $S$ would, is an extensive bottom-land country, which ity to carry off the local surplus water, and aid in relieving the Mississippi whenever a threatened over-
flow at that part of its course created a call for an extra outlet.

Between Omaha, Neb., and St. Joseph, Mo., a good deep and broad canal would aid greatly in preventing

At Kansas. City, too, relief from $_{t}$ inundations, of the lower portions of that city can be, secured by one or surplus water whenever a surplis approaches with all its threatened consequences.

On the middle and upper Ohio, along the rivers, of Kansas, wherever there is a necessity in localities mentioned or others not named; wherever are found large rivers or threatening streams subject to extraordinary floods, in any part of the world, this proposed
plan will bring security from ordinary ensving disasplan will bring security from ordinary ensing disas-
trous consequences. Think for a little how much this means.

From the northwest corner of Lake Pontchartrain a cypress swamp extends north for many miles. This greatest part of the nearly all over its surface, for the become farm land of great value on account of its fertility. This can all be drained by one canal and a
levee along the shore of the lake, then pump the water
over the levee into the lake.

over the levee into the lake.
Many other similar localities can be reclaimed in Many other similar localities can be reclaimed in
like manner. The above proposed system is of vast like manner. The above proposed system is of vast investigated at the earliest possible opportunity volves, and whose duty it is to have this work of altogether unprecedented importance performed.

Whose duty should it be, if not the unbounded duty of the people, all the people, of the great republic, the United States of America? Every citizen of this whole country should be interested. This work will
all of them, both individually and collectively.

Spokane, Wash., November 2, 1908.

From the returns compiled by Lloyd's Register, it appears that, excluding warships, there were 319 vessels 